\title{
Dynamics and hypotheses of gene order shifts in mitochondrial genomes of Baikalian amphipods
}

\author{
Elena A. Sirotinina \\ Lab of gene systematics \\ LIN SB RAS \\ Irkutsk, Russia \\ haleo.inc@gmail.com
}

\author{
Dmitry Yu. Sherbakov \\ Lab of gene systematics \\ LIN SB RAS \\ Irkutsk, Russia \\ dysh007@gmail.com
}

\author{
Elena V. Romanova \\ Lab of gene systematics \\ LIN SB RAS \\ Irkutsk, Russia \\ elena_romanova@lin.irk.ru
}

\begin{abstract}
In our study we describe the variabilities in the mitochondrial gene order of amphipod species. Using phylogenetic inference and the methods of gene order rearrangement counting we identify species with mostly rearranged mitochondrial genomes and try to explain the reasons for such variability.
\end{abstract}

Keywords — Lake Baikal; mitochondrial genomes; amphipods; gene rearrangement

\section{Motivation and aim}

Motivation

Gene order in the mitochondrial genomes (mt genomes) of invertebrates is shown to be different. The dynamics of such changes are usually studied by analysis of different organisms' lineages. It is notable that in some animal lineages the mitochondrial gene order maintained the same for the long periods, whereas other lineages demonstrate a significant variation of this feature [1]. Amphipods can be a good model for studying mitochondrial gene order dynamics because of a relatively large number of species with sequenced $\mathrm{mt}$ genomes (about 100) available and due to the variability of their $\mathrm{mt}$ gene order. Recent studies showed that endemic amphipods from Lake Baikal possess rearranged $\mathrm{mt}$ genomes in comparison to the non-Baikalian species $[1,2,4]$.

Aim

Amphipod species with currently sequenced mt genomes belong to different taxa and occupy different ecological niches, which makes it possible to assess whether mt gene order rearrangements correlate with ecological features of species, their life-history traits, time of lineage divergence, etc. For instance, there was shown a positive correlation between the rate of gene rearrangements with the level of nucleotide substitutions in their protein-coding genes [5, 6]. We also recently showed the significant acceleration in mutation rate in both lineages of Baikalian amphipods in comparison to species from gen. Gammarus [7].

There are two mechanisms of the new gene orders emergence: 1) an erroneous duplication of the whole $\mathrm{mt}$ genome sequence during replication by "rolling ring" mechanism, associated with the DNA polymerase slippage on specific sites or repeats, and 2) a partial tandem duplication and random loss (TDRL) of a certain genome region [8]. To measure such gene rearrangements quantitatively it is necessary to determine the number of mutational steps (permutations) from the ancestral gene order pattern to ones for the modern species. An ancestral gene pattern for any lineage is identified using phylogenetic reconstructions.

\section{Methods}

We defined an ancestral $\mathrm{mt}$ gene order pattern for all amphipods performing phylogenetic inference based on amino acid sequences of $13 \mathrm{mt}$ protein-coding genes. To estimate possible scenarios of gene order changing, including the minimum number of elementary gene permutations, we used CREx and SORT2 [9, 10]. In some cases we assessed the changes manually by taking the number of displaced genes into account individually, and also counting the number of moved gene blocks grouped based on the proximity of their location.

\section{Results}

The analysis showed that Pancrustacean pattern (the pattern common for all crustaceans and insects) generally used as a reference in $\mathrm{mt}$ gene rearrangements studies [1, 3] differ from deduced Amphipoda pattern by the location of some tRNA genes. The Amphipoda pattern deduced in our study turned out to be the same as one for gen. Gammarus species. The phylogenetic analysis showed that this pattern was ancestral for both lineages of Baikalian amphipods. Thus the rearranged patterns of the majority of modern Baikalian amphipods were acquired during the evolutionary process in Lake Baikal. Notably, Baikalian amphipods from the second lineage (the one includes the majority of species which have very divergent morphological and ecological traits) possess gene order alteration affecting mainly tRNA gene positions. Whereas amphipods from the first lineage, which comprise much less number of species, having a smooth body and inhabiting mainly in shallow water, have much more complex alteration in their $\mathrm{mt}$ gene order, affecting the positions of the protein-coding and ribosomal genes [2]. We hypothesize that the fixation of highly rearranged $\mathrm{mt}$ genome becomes possible due to relaxed purifying selection in populations with low genetic diversity and low estimated effective population size. The shallow-water amphipod species in Lake Baikal could probably be more prone to the dramatic decrease of their population and consequently genetic diversity due to environmental changes in the Lake.

\section{Acknowledgment}

The work was supported by the governmentally funded project 0345e2019e0004 (AAAA-A16-116122110060-9). 


\section{References}

[1] F. Kilpert, L. Podsiadlowski. "The complete mitochondrial genome of the common sea slater, Ligia oceanica (Crustacea, Isopoda) bears a novel gene order and unusual control region features", BMC Genomics, vol.7, p.241, 2006.

[2] E. V. Romanova, V. V. Aleoshin, R. M. Kamaltynov, K V.Mikhailov, M. D. Logacheva, E. A. Sirotinina et al. "Evolution of mitochondrial genomes in Baikalian amphipods", BMC genomics, vol. 17 , pp. 1016, 2016

[3] L. Krebes, R. Bastrop. "The mitogenome of Gammarus duebeni (Crustacea Amphipoda): A new gene order and non-neutral sequence evolution of tandem repeats in the control region", Comp Biochem Physiol, vol.7(2), pp.201-11, 2012.

[4] Y. Shen, Q. Kou, Z. Zhong, X. Li, L. He, S .He, X. Gan. "The first complete mitogenome of the South China deep-sea giant isopod Bathynomus sp. (Crustacea: Isopoda: Cirolanidae) allows insights into the early mitogenomic evolution of isopods", Ecol Evol., vol.16, pp. 1869-1881, 2017.

[5] R. Shao, M. Dowton, A. Murrell, S. C. Barker. "Rates of gene rearrangement and nucleotide substitution are correlated in the mitochondrial genomes of insects", Mol Biol Evol, vol.20(10), pp.1612-1619, 2003.
[6] S. Fourdrilis, A. M. de Frias Martins, T. Backeljau. "Relation between mitochondrial DNA hyperdiversity, mutation rate and mitochondrial genome evolution in Melarhaphe neritoides (Gastropoda: Littorinidae) and other", Sci Rep., vol. 8 (1), p.17964, 2018.

[7] E. V. Romanova, D. Y. Sherbakov. "Different rates of molecular evolution of mitochondrial genes in Baikalian and non-Baikalian amphipods." Limnology and Freshwater Biology, vol.6, pp.339-344, 2019.

[8] Y. Xia, Y. Zheng, R. W. Murphy, X. Zeng. "Intraspecific rearrangement of mitochondrial genome suggests the prevalence of the tandem duplication-random loss (TDLR) mechanism in Quasipaa boulengeri", BMC Genomics, vol.17, p. 965, 2016.

[9] M. Bernt, D. Merkle, K. Ramsch, G. Fritzsch. "CREx: Inferring Genomic Rearrangements Based on Common Intervals", Bioinformatics, vol. 23(21), pp.2957-2958, 2007.

[10] Y. L. Huang, C. C. Huang, C. Y. Tang, C. L. Lu. "SoRT2: a tool for sorting genomes and reconstructing phylogenetic trees by reversals, generalized transpositions and translocations", Nucleic Acids Res, vol.38, pp.221-227, 2010 